\title{
Molecular characterization of the genes encoding acetohydroxy acid synthase in the cyanobacterium Spirulina platensis
}

\author{
A. Milano, E. De Rossi, E. Zanaria, L. Barbierato, O. Ciferri and G. Riccardi* \\ Dipartimento di Genetica e Microbiologia 'A. Buzzati Traverso', Via Abbiategrasso 207, 27100 Pavia, Italy
}

(Received 3 January 1992; revised 10 March 1992; accepted 16 March 1992)

\begin{abstract}
The enzyme acetohydroxy acid synthase (AHS), which catalyses the first common step in the biosynthesis of isoleucine, leucine and valine, has been demonstrated to be present in Spirulina platensis in two isoenzymic forms. The complete nucleotide sequences of the genes $i l v X$ and $i l v W$ encoding these two enzymes have been determined. Sequence analysis revealed the presence of two open reading frames, of 1836 and 1737 nucleotides for $i l v X$ and $i l v W$, respectively. The predicted amino acid sequences of the two isoenzymes, compared with the Synechococcus PCC 7942 AHS enzyme and the large subunits of the Escherichia coli AHSI, II, III isoenzymes, revealed a notable degree of similarity. A small subunit has not been identified for either of the $S$. platensis AHS isoenzymes. Analysis by Northern blot hybridization demonstrated that the $i l v X$ and $i l v W$ genes are transcribed to give mRNA species of approximately $2 \cdot 15 \mathrm{~kb}$ and $1.95 \mathrm{~kb}$, respectively.
\end{abstract}

\section{Introduction}

The study of amino acid biosynthesis, which has substantially contributed to our present knowledge of microbial metabolism, has been very limited in cyanobacteria (Riccardi et al., 1989). We are studying the pathway of isoleucine, valine and leucine biosynthesis in Spirulina platensis as an approach to understanding the regulation of cyanobacterial metabolism (Riccardi et al., 1990). Acetohydroxy acid synthase (AHS, EC 4.1.3.18) is the first common enzyme in the biosynthetic pathways to valine, isoleucine and leucine. AHS has recently been demonstrated to be the target of two new and structurally unrelated classes of herbicides, the sulphonylureas (Chaleff \& Mauvais, 1984; Ray, 1984) and the imidazolinones (Shaner et al., 1984).

Three AHS isoenzymes have been found in Escherichia coli and Salmonella typhimurium (De Felice et al., 1982; Umbarger, 1983). The genes encoding these isoenzymes are organized in operons and include ilvBN,ilvGM and $i l v I H$, coding for isoenzymes I, II and III, respectively

\footnotetext{
* Author for correspondence. Tel. (0382) 391574; fax (0382) 528496.

Abbreviations: AHS, acetohydroxy acid synthase; RBS, ribosomebinding site.

The nucleotide sequence data reported in this paper have been submitted to GenBank and have been assigned the accession numbers M75906 for ilvX and M75907 for $i l v W$, respectively.
}

(Wek et al., 1985; Lawther et al., 1981; Squires et al., 1983). In these bacteria AHS is a tetramer composed of two large and two small subunits. The large subunits have a molecular mass of about $60 \mathrm{kDa}$, while the small subunits have molecular mass of 10 to $20 \mathrm{kDa}$ (Eoyang \& Silverman, 1988; Schloss \& Van Dyk, 1988; Barak et al., 1988). Only one gene encoding functional AHS lacking a small subunit was isolated from yeast (Falco \& Dumas, 1985; Falco et al., 1985). Genomic clones encoding AHS have been isolated and sequenced from the higher plants Arabidopsis thaliana and Nicotiana tabacum (Mazur et al., 1987). In $A$. thaliana the gene codes for an AHS of molecular mass $72593 \mathrm{Da}$. Friedberg \& Seijffers (1990) isolated and sequenced a gene from Synechococcus PCC 7942 that encodes a protein of 612 amino acids showing high sequence similarity with the AHSIII large subunit of $E$. coli and the monomeric tobacco AHS.

Two isoforms of AHS were detected in cell-free extracts of Spirulina platensis and separated both by ionexchange chromatography and by hydrophobic interaction (Forlani et al., 1991). The isoforms differ in $\mathrm{pH}$ optimum, FAD requirement for both activity and stability, and heat lability (Forlani et al., 1991). The corresponding genes, ilv $X$ and $i l v W$ (named $i l v Y$ in our previous papers), were isolated from a $\lambda$ library of $S$. platensis genomic DNA. The $4.2 \mathrm{~kb} C l a \mathrm{I}(i l v X)$ and the

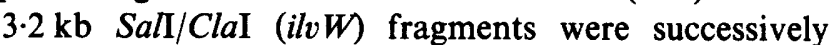
subcloned in the plasmid vector pAT153 (Maniatis et al., 
1982). The ilvX gene was able to complement a suitable mutant of $E$. coli while the ilvW gene supported poor growth of the same E. coli mutant (Riccardi et al., 1991).

We here present the complete nucleotide sequence and a preliminary transcriptional analysis of the two genes (ilv $X$, ilvW) encoding AHS isolated from S. platensis. We also compare the deduced amino acid sequences of the two AHS isoenzymes to that of the large subunits of the $E$. coli AHS isoenzymes I, II and III and to the Synechococcus PCC 7942 enzyme.

\section{Methods}

Culture conditions, $R N A$ isolation and Northern hybridization. Culture conditions for $S$. platensis $\mathrm{Cl}$ have been previously described (Riccardi et al., 1981). RNA was extracted from 1 litre of $S$. platensis culture grown to mid-exponential phase and then harvested by filtration on paper discs. Cells were immediately frozen in liquid nitrogen, ground using a ceramic mortar and pestle, and resuspended in guanidine. $\mathrm{HCl}$ buffer ( $8 \mathrm{M}$-guanidine. $\mathrm{HCl}, 0 \cdot 1 \mathrm{M}$-sodium acetate, $5 \mathrm{mM}$-dithiothreitol, $0.5 \%$ sodium lauryl sarcosinate, $\mathrm{pH} 5 \cdot 2$ ). The cell suspension was extracted with an equal volume of water-saturated phenol and the aqueous phase was re-extracted with phenol/chloroform/isoamyl alcohol prior to a final extraction with chloroform/isoamyl alcohol (Maniatis et al., 1982). Sodium acetate (pH 5.2) was added to a final concentration of $0.3 \mathrm{M}$ and RNA was precipitated by adding 2.5 vols ice-cold ethanol followed by storage at $-20^{\circ} \mathrm{C}$ for at least $2 \mathrm{~h}$. RNA was recovered by centrifugation at $10000 \mathrm{~g}$ for $10 \mathrm{~min}$ at $4^{\circ} \mathrm{C}$. The pellet was washed with ice-cold $70 \%(\mathrm{v} / \mathrm{v})$ ethanol, recentrifuged briefly, and redissolved in a small volume of water. Three volumes of ethanol were added, and the preparation was stored at $-70^{\circ} \mathrm{C}$ until needed.

Aliquots containing $20 \mu \mathrm{g}$ of the RNA were fractionated by formaldehyde-agarose gel electrophoresis (Maniatis et al., 1982) and transferred by an alkali blotting procedure to Hybond- $\mathrm{N}^{+}$filters (Amersham) according to the manufacturer's instructions. Filters were prehybridized and hybridized according to standard procedures (Maniatis et al., 1982). The hybridized blots were washed twice for 10 min each in $2 \times \mathrm{SSC}\left(\mathrm{g} \mathrm{l}^{-1}: 17.5 \mathrm{NaCl}, 8.8 \mathrm{Na}_{3} \mathrm{C}_{6} \mathrm{H}_{5} \mathrm{O}_{7} .2 \mathrm{H}_{2} \mathrm{O}, \mathrm{pH} 7 \cdot 2\right)$ and $0.1 \%(\mathrm{w} / \mathrm{v}) \mathrm{SDS}$ at $50^{\circ} \mathrm{C}$ and exposed to X-Omat AR X-ray films at $-70^{\circ} \mathrm{C}$ with an intensifying screen.

DNA sequence determination. Double-strand nucleotide sequencing of the two ilv genes from $S$. platensis was performed on both strands by the dideoxy-chain termination method (Sanger et al., 1977), after subcloning overlapping restriction fragments, ranging from 200 to $500 \mathrm{bp}$, in the plasmid pGEM4Z (Promega Corp.). A sequencing kit was used (Pharmacia, LKB); reactions were carried out according to the manufacturer's instructions. SP6 and T7 promoter sequences (Promega Corp.) were used as primers. To overcome secondary structures in G + C-rich stretches, 7-deaza-dGTP was used rather than $\mathrm{dGTP}$ in the sequencing reactions. DNA sequences were analysed with the DNA and Protein Sequence Analysis Programs of D. W. Mount and B. Conrad, University of Arizona. Protein sequence alignments were generated with the CLUSTAL program (Higgins \& Sharp, 1989). Possible secondary structures downstream of $i l v$ genes were identified using the RSEC program (Lang \& Burger, 1986). Free energy of formation for these structures was also calculated using the RSEC program.

\section{Results and Discussion}

\section{Sequencing of the S. platensis genes encoding AHS}

In a previous study, a $S$. platensis genomic library was shown to contain a $4.2 \mathrm{~kb}$ ClaI fragment and a $3.2 \mathrm{~kb}$ ClaI/SalI fragment carrying the presumptive genes encoding AHS (Riccardi et al., 1991). The sequences of these two genes and of their deduced polypeptides are shown in Fig. $1(a, b)$. A total of 3373 and 3170 nucleotides including respectively the entire ilv $X$ and $i l v W$ genes and their $5^{\prime}$ and $3^{\prime}$ flanking regions were sequenced. The coding region of $S$. platensis ilv $X$ includes 1836 nucleotides, corresponding to 612 amino acids, while that of the ilvW gene has 1737 nucleotides, corresponding to 579 amino acids. The molecular masses predicted by the sequences are about $67 \mathrm{kDa}$ for $i l v X$ and $63 \mathrm{kDa}$ for ilvW. In ilvX, GTG appears to be the initiation codon. The same codon was found in the corresponding gene of Synechococcus PCC 7942 (Friedberg \& Seijffers, 1990), as well as in other cyanobacterial genes (Reddy et al., 1988; Yasui et al., 1988). GTG is also a translation start codon in other organisms (Kozak, 1983). Each gene is preceded by a polypurine tract which resembles the sequences proposed by Shine \& Dalgarno (1974) for a ribosome-binding site (RBS). The ilvX gene is preceded by the sequence 5' GAGGAG 3', 6 to 11 nucleotides upstream from the apparent translation start codon. The ilv $W$ gene is preceded by the sequence 5' GAGGAA 3', 5 to 10 nucleotides upstream from the apparent translation start signal. The sequence of the $16 \mathrm{~S}$ rRNA from the cyanobacterium Anacystis nidulans (Tomioka \& Sugiura, 1983) is very similar to that of the 16S rRNA from $E$. coli, suggesting that analogous RBS sequences are likely to occur in both organisms. Indeed, sequences such as AAGG, GGAG have been found upstream from most of the cyanobacterial genes sequenced so far (Tandeau de Marsac \& Houmard, 1987).

AHS in other bacteria studied is composed of two large and two small subunits whose genes are organized in

Fig. 1 (on following five pages). Nucleotide and deduced amino acid sequences of the $i l v X(a)$ and $i l v W(b)$ genes. Nucleotides are numbered starting from the HincII site for $i l v X$ and from the SalI site for $i l v W$ (Riccardi et al., 1991). A Shine-Dalgarno box and potential promoter regions are indicated by underlining. Palindromic sequences in the $3^{\prime}$ non-coding region are indicated by arrows below the sequence. The dyad symmetry found in the upstream sequences of $i l v X$ and $i l v W$ is indicated by paired arrows above the sequence. 
(a) Sequence of il:X

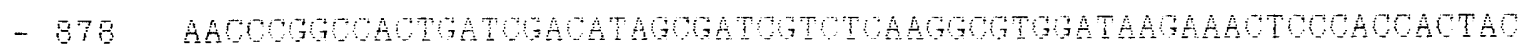

- 16

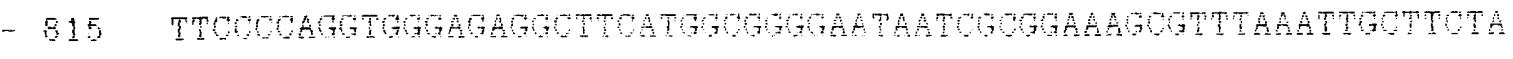

$-755$

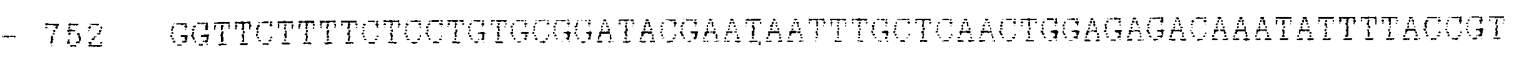

$-60$

$-68$

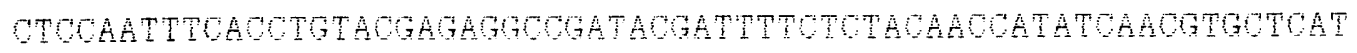

$-E 7$

$-626$

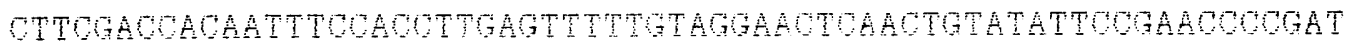

$-564$

$-603$

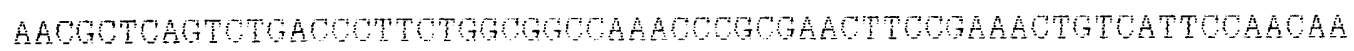

$-501$

$-5010$

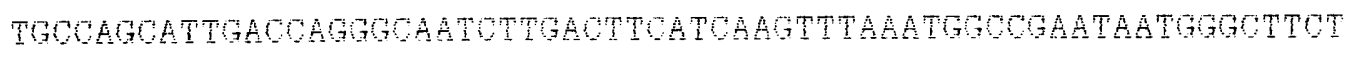

$-40$

$-437$

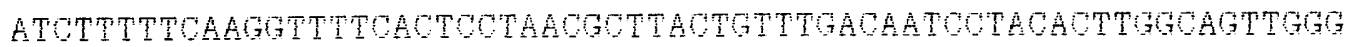

$-375$

$-\quad 374$

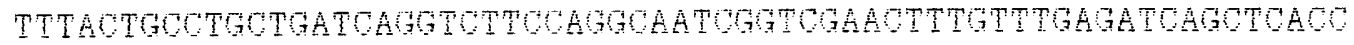

$-312$

$-811$

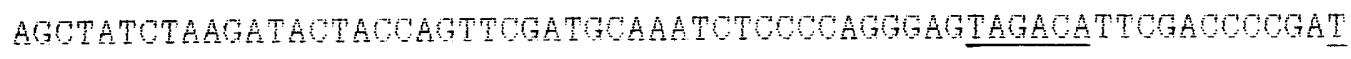

$-249$

$-748$

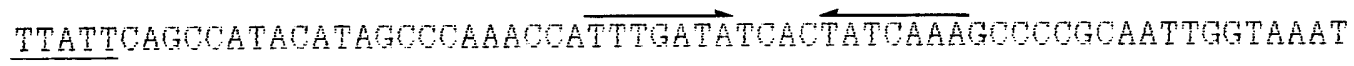

$-185$

CGTGATACAATTGAGOAAAOUYGAOCOCTAAOOTATOGGOOCAOCOTTAOTTAT

$-186$

$-121$

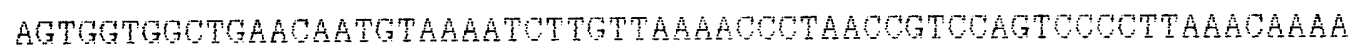

$-12-$

TOCTTATOTAAATAGAGGTTTABOTAACAATATAOTAAATTAGAGGAGOGACGTGCAA

M G

CTTCAAAOTAAATTGCTGOCAAGOGTGOACAOGTGOGTTOOOOTOATTGATAGTOTAAA L $Q T E$ T I A A K F A T G A E E I I D G L

443 GCTCGCATTGTCGCAGAAGCCTTCCATATTGCCAGCACAGGCOGTCOCGGTOCGGTACTCATA 
700 CATGCAGAAATGCGGAATTGGCAGAGCTTTTTCAGATTCOGGTAAOTAOCACATTAATGGGG $\begin{array}{llllllllllllllllllllll}H & A & E & I & A & E & L & A & E & L & E & Q & I & F & \vee & T & T & T & L & M & G\end{array}$

589 CGCGTACOGGGAAOTTGATAATTTGATOTAGGGOGAAGGTGATTCATATTGAOATGAO

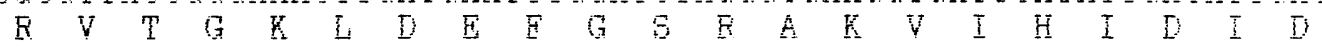

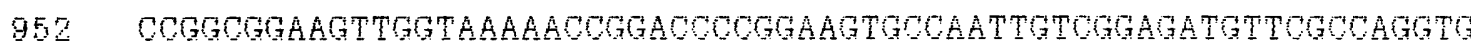
P A E V G $F$ V $E$ T $F$ E V $F$ I V G D V E $Q V$

1015 TTGATACACOTGTTGCGOCGOTGCOGAGAAATTGAGATGTOGGGAAOGATAATCAAAOTCAA

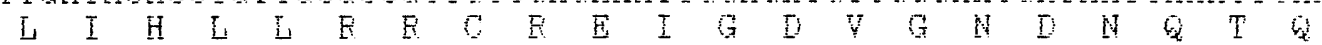

1073 TOGTGGTAGAGCGGATTAACGOTGGOCGGAAGATATCOTOTGGTGGTOCOTOTTATTOT

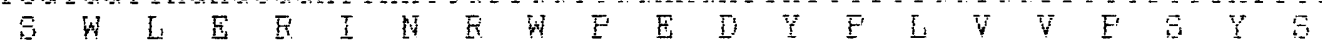

1141 GATAGCOTOTOTCOCOAAGAGTGATOGCAGAATTAGGAAAAATGOCAOCGGATGGOTAOTAT

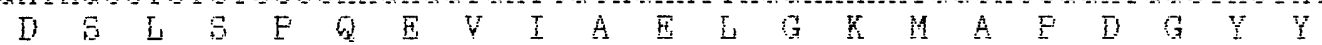

1204 ACCACGGATGTGGGTCAGCACCAAATGTGGGCGGOTOAATTTTGAAAATTGCOCOCGTOAG

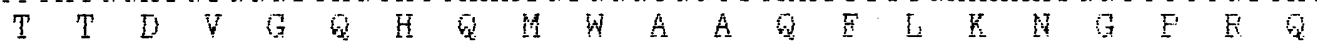

1267 TGGATATCTAGCGCTGGGTTAGGAOTATGGGCTATGGTATCOCGTCGGCTATGGGAGCTAAA

1392 CAAGAACTOGGAACCATAGCACAATATGGCATAAATGTCAAGAOCOTAATTATTAATAAOGGT

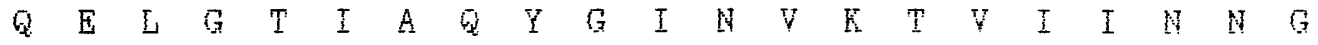

1456 TGGCAAGGTATGGTOCGCCAGTGGCAACAAGCOTTTATGGTGAGOGTTATTCGGOTTCTAAC

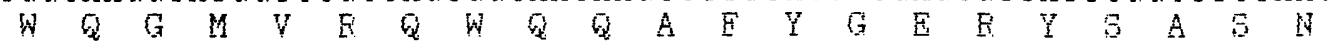

1518

1519 ATGGAAATCGGAATGCCAGATTTTGAAATGTTAGCACGTTCOTATGGGGTAAAAGGCATGGTC

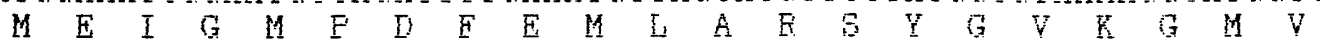

1581

1582 GTTAGAGTCGTGACGAATTACACCAAGCACTAGCAGAAATGTTGGCTTATGATGGTCOTGTG

1644

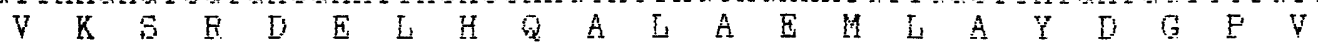

1645 CTGATGGATGTTCATGTACOAAGGAGAAATTGTTATOCTATGOTAGCACCAGGAAGGAGT

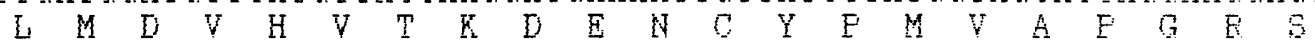

1708 AATGCTCAGATGATAGGATTACCTGAACGCOGCCAGTTAGAAAAAGCGGTTGGTTAATOTAT

1770

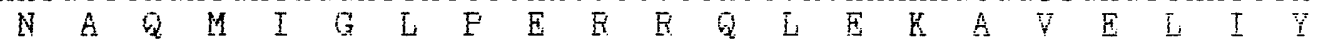

1771 TGCAGTAATTTGGCGOGAAAAATGTOGCGAGTAACAATTTOTGTOCTGAGTGTGGCAOTAAA

1833

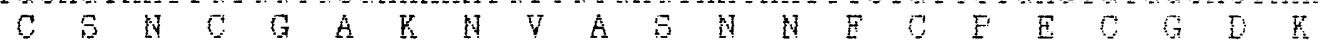

1834 CTATAAACACTOTOTATTACOTGTCAGAOATCAGGATGATTGGTGTGOTTTGTTAGT L

1896

1897 GATAAGTAGAGACAGGGTTGTCTTGTCTCTACTGTGTCAATTOTCGATGAGGGGTATGOTG

1959

1960 ACACCTAGCAGTCAATTAATAGGGAGAAGCGGTTACCTCOCGTOGGAATAGAAAATCAGAT

2022

2023 GGTCAGCCAAATCTGGATTTTTAAGAGTAGCTGATATTGGTGGGTGCAAAOTTAATCAOTI 
2086

CGTAATCAAGAGTTTT TAGGAATTTTTCGGCCAGGGTAAAAAGGAAGTTTCGGGTTAGAOA

2148

2149 AGTTGATTGATAAATCTCTGGGGTATCTGTCTAATTAAATTGTATOGTCOAATTOCOTA

2211

2212 AATTATGGTGCATGACTGTOTGAATGTTATGGTGGTAATTGACCOTTTOAAAGGGTOGT

2274

2275 CACCGGGTTCGCGATTAAATGCGATCGCATATTTTTCACOTCOCOCAAATAGATACAAACCOT

2337

2338

GTATTAAACCGGTCCGATAAATATAAGCACCOCGATAATTIATCAGGAATAGCAGTAATAATAG

2400

2401 CGGGGAGATTAGGGTGATCTTGTAACATTCOCAAAGAATATTTGAGAAATGGAACCAGCOA

2464 CCCGCCAATTTGGAACAAAGATTGAACAGTTAAC

(b) Sequence of ilvW

$-522$

$-459$

$-396$

$-333$

$-270$

$-207$

- 144

- 81

$-18$

47

110

17.

236

299

362

425

488

TCGACCTGGCGATGCAACGGATCGCGAACTTTAGAAGTAGCOGAAGOGTAACGGAOCOGTOTC CGATCCTTAGCACGCAGCOTTGCGAAAGCOCTGCAAAATTTCTACOCAGAAATAGCOGAATTT CAAGTGCGCGATTATAAAGTGCGGATTTTAGATGGTAAAGCOGGAACTTCGTCOAAAACTCGT GTATTGGTAGAGTCTAGTAATGGTCATCAGCGTTGGOTAOCGTAGAGTCTTTACTAACATCT TAGAGCOTACOTATCAAGCOGTGOTAGAAGGTTAGAATATGGCTTAATGTTGCAAACAGCAA ACCOCATCCAATTCAGTTATATTAGCGGATCCTCACTGTAAATAGGAGTGATAATCAGCTA GGGATGGCATTATTGGCATAGCATAAAATGGCAATAATGGCAAGATATGAATCAGAATTGT CACAAATATAAAAAATTGATGACA TTGOTCAGGTAATCCCAACOATAATTTATCOTAGGCAT CAGAGATGGAGATGTTATGCAGGACCAAAAACAAGCOGTAAAAAGAGTAACAGGAGOCTTTG

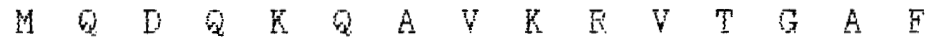

CCOTGATTGATAOCOTGCGCOGCOACGGTGTGCAGCATATTTTTGGOTATCCAGGAGGTTCAA

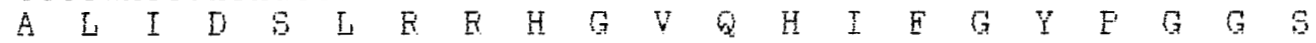

ACTTACOCATTACGACGAAATATATAGACOCOAACAAGOCGGGGAAATCAAACATTATOTGG $\begin{array}{llllllllllllllllllllll} & \mathrm{L} & E & I & Y & D & E & I & Y & E & A & E & G & A & G & E & I & F & H & Y & L\end{array}$

TGCGGCACGAACAAGGAGCGGCCCACGOTGCOGATGGATATGCOCGGAGTAOCGGAAAGGTGG

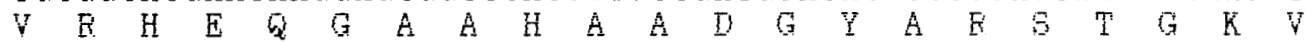

GGGTATGTOTAGCOACTTOTGGACOGGGAGCACCAATTAGTGACAGGTOTAGCCACTGGTT

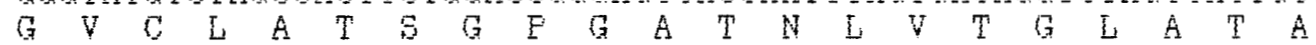
ATCTCGATTCTGTCOCGGTGTTAGCOATTAOCGGACAAGTCCOCOGCAGCGOCTTGGGAACAG

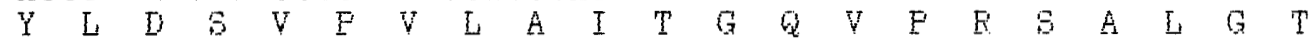
ATGCGTTTCAGGAAATRATATTTTTGGGATTACCOTACCOATTGTTAAACATTCOTATCTAG

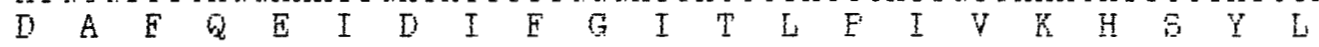
TCCGCGAACCTTCGGAATTACCGAGAATTGTGGTCGAGGCATTTOAOCTAGCOATGAGTGGCA $\begin{array}{lllllllllllllllllllll}V & B & E & P & G & E & L & F & E & I & V & V & E & A & E & H & L & A & M & G & G\end{array}$

GACCGGGACCTGTGTTAATGACATTCOCAAAGATGTGGGTAATGCTCAAATGATTATATTC $\begin{array}{llllllllllllllllllllll} & F & G & F & V & I & I & D & I & F & \mathbb{I} & D & V & G & N & A & G & I & D & Y & I\end{array}$

551 CGGTAGAACCAGGGTCAGTCAGACGGGTGGGATATCGTCCCACGGAGCGGGGAAATCCTCGAC

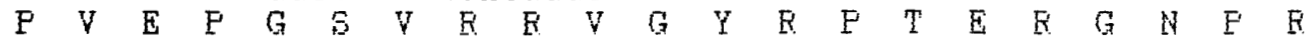

$-460$

$-397$

$-.334$

$-271$

$-208$

$-145$

- 82

$-19$

46

109

172

235

298

361

424

487

550

613 
614 AAATTAATCAGGCTTPACAGTTGATTTCCGAAGCTACTAAACCCCTCOTATATGTGGGGGGAG 676

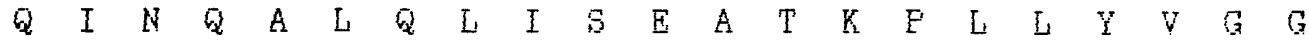

677 GTGCAATTATGGCGGGGGCTCATGCTGAAATCGCCGAATTGTOGGAACGCTTCCAAATACCAG $G \quad A \quad I \quad M \quad A \quad G \quad A \quad H \quad A \quad E$ I $A$ E

740 TTACTAGCACTTTGATGGGAAAAGGTCGTTTGATGAAAATCACCCCOTATCTOTGGGAATCG $\begin{array}{lllllllllllllllllllll} & T & T & T & L & M & G & K & G & F & E & D & E & N & H & F & L & G & L & G & I\end{array}$

303 TGGGAATGCTGGGAATGCACGGACCGCCTATGCTAATTTTGCGGTGATGGAATTGGATTTCG

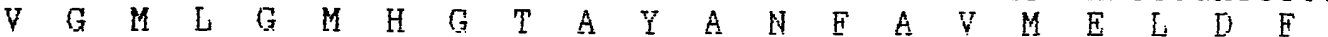

366 TGATCGCGGTGGGGGTTCGCTTTGATGACCGGGTGGCGGGTACTGGAGATCAATTCGCOCATA $\begin{array}{lllllllllllllllllllll} & I & A & V & G & V & E & E & D & D & F & V & A & G & T & G & I & Q & E & A & H\end{array}$

929 GCGCTAAGGTGATTCATATTGATATTGACCOGGCGGAAGTGGGCAAAAATCGCAGTACTGATG $\begin{array}{llllllllllllllllllllllll}5 & A & K & V & I & H & I & D & I & D & F & A & E & V & G & K & N & E & G & T & D\end{array}$

992 TTCCTATTGTGGGAGATGTGCGACAGGTTTTGGGGGATATGCTACAACGTACGTATCATTGGG

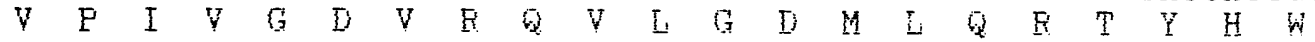

1055 AACGGAAATAAGCOGGAACAAACCOAGGAATGGCAGTGAOCTTAATCAATTGCGTGAACCAA

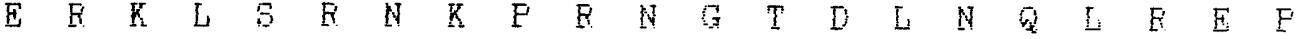

1118 TACCOCTGACGGTTOCTCACOOGGAAGATGGTATOTCTCOTCAAGATGGTGATTGGGAACTTA

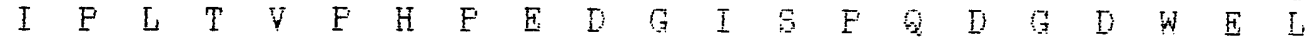

1181 GCCATCAATGTCOTGATGCTTTCTATACTAOTGATGTGGGTCAGCATOAAATGTGGGCGGGCO $\begin{array}{lllllllllllllllllllll}S & H & Q & C & F & D & A & E & Y & T & T & D & V & G & Q & H & \square & M & V & A & G\end{array}$

1244 AATTCGTOCAAAATGOCOCCCGACGTTGGATGAOTAGOGGOGGTTTAGGGACTATGGGTTACG

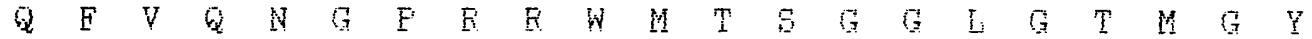

1307 GTTTACOTGCTGOTGTGGGGGTTAAGTGGCTCATCOTCATGATACGGTTACCTGTATTAGGG

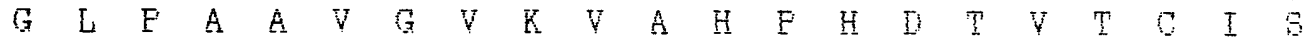

1370 GTGATGGTAGTTCCAAATGAATATGCAAGAATTGGGAACTATTGOTAGTATGGTATTGGGG

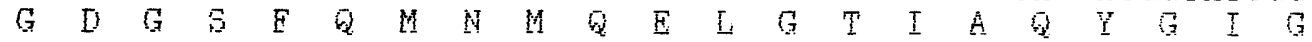

1431 TTAAGGTGATTATTCTCAATAATGGOTGGTTGOGAATGGTGCGCCAGTGGCAACATATGTTTT

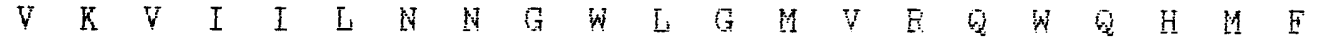

1496 ATAATGAOCGCTATGAGGCTACTAACOTGGAAGATGGAACCOCAGAATTYGOOOGGTTAOCTG

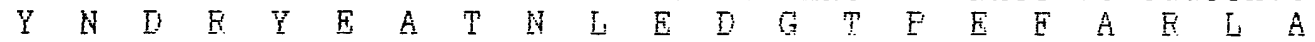

1559 ATGTTTATGGACTTGAGGCTATGAACGTCOGCOAACGGAAGATTTACCAAAGGCGACTGCOTA

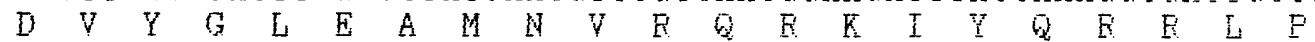

1621

1622 AAGCTCTATCTCACAAGGGTCCGATGATCOTAGATGTTCGGGTTACOCGTGATGAGGAOTGTT K $A \begin{array}{llllllllllllllllllll} & A & G & H & K & G & F & M & I & L & D & V & F & V & T & E & D & E & D & C\end{array}$

1684

1685 ACCCGATGGTAGCCCCCGGTCACGATAACAGCGACATGATGGGTTTATCCAGTTAGTTGCTOT $\begin{array}{llllllllllllllllll}Y & F & M & V & A & F & G & H & D & N & G & I & M & M & G & L & 5 & B\end{array}$

1743 AACCCCTCACCCCCCCTTAGTGCTTTATGGGGGGGTATTOTGTCTGAGAGCAAATCTTACATT

1811 AACCTGAAAAAACGCCATATTGATTTACAAAAATTCGCAAAAATTAATAATGTTTTGTTTGAT 1873

1874 TAATCAGGTGTAAAATTGGTGACAAAATATCOTAAATTAAAATGGTAGTTCACTCTCAATCCC 1936

1937 TAACCGGAATGATCTATCCTAATATGAGCCGGAGACTAAGGGAAATCGAGAGGAGCAAAGTCO 1999 


\begin{tabular}{|c|c|}
\hline 063 & AAAAATCACAATTGOCACCGTGTTATAATTATTGATTTATCOGGTTTCAGGAGATOGTCAGA \\
\hline 126 & TGACAAATAATGTTGCATCAGAAAACCGTAATGTCGCGATTGTCGOCOCOTATTTGAGOCGCA \\
\hline 2189 & AAACAGCGOTGCTAGAAAGTOTGCTGAAAGCGACGGGAACGATTACOCGTAAAGOTACGOCTA \\
\hline 2252 & AAGAAGGCATCGGATCGCGGATAGCGTACTGGAGGOTOGCGATCGCAATATOAGTOTAOAAB \\
\hline 8915 & TCAATGCOCGOTTATCATGAATATGGCGGCATTACATTTOACTCOTOGCATOTCCAOGTTOG \\
\hline 2379 & GTAGATATTGCOCAAGACACATTACAACGCCOTGTAGGGTGCAGGTTAGCATAAGTAGTTTG \\
\hline 441 & TGCOAAGCOCAATTACAGOTAATTTTTGGATGATTGGGAAATTCOCATATMGTGTMATTAA \\
\hline 3504 & TAAAATGGATCGGGCTTCTOTGATGAAGAAGGCAGCTCCOTCAACTTTTAGAGTTATAAA \\
\hline 567 & TGCOOTGAAAACTGTATCGAOTCGCCCCATAGTTCCCCAAGAATATCCGATCGCOAAAGCTTC \\
\hline 63.30 & AGAATTAATTGGTTTTATOG \\
\hline
\end{tabular}

operons (Umbarger, 1983). Attempts to find additional open reading frames in the $3^{\prime}$ flanking regions of both $i l v X$ and $i l v W$ were unsuccessful. We hypothesize that the AHS small subunit either is not present in $S$. platensis, similar to the situation in Arabidopsis thaliana and Nicotiana tabacum (Mazur et al., 1987), Brassica napus (Wiersma et al., 1989), yeast (Falco et al., 1985) and Synechococcus PCC 7942 (Friedberg \& Seijffers, 1990), or is not organized in an operon with ilvX or ilvW. However, the existence of a small subunit in the $S$. platensis AHS isoenzymes cannot be excluded. We intend to purify the $S$. platensis ilv $X$ and ilv $W$ gene products from recombinant $E$. coli and to prepare antibodies against them in order to purify the isoenzymes from $S$. platensis by affinity chromatography. This approach could further elucidate the subunit structure of the cyanobacterial AHS.

\section{Amino acid sequences encoded by ilvX and ilvW}

The deduced amino acid sequences of these genes can be aligned with Synechococcus PCC 7942 and E. coli AHS proteins as shown in Fig. 2. The six AHS isoenzymes show close amino acid sequence similarity. Previously, a comparison of the ilvG, ilvB and ilvI gene products revealed three regions of similarity (Wek et al., 1985). These three regions of similarity can also be identified in the $i l v X$ and $i l v W$ gene products. The overall degree of similarity is not equally distributed over the entire lengths of the six polypeptides: several of these blocks extend for 20 to 30 amino acids with few mismatches and other regions show much less similarity. However, the degree of overall identity indicates that these genes encode a family of related polypeptides. In addition to these regions there is conserved similarity between the carboxyl termini of the $i l v X$ and $i l v W$ gene products and the ilvB and $i l v G$ gene products that is not shared with the ilvI product.

Comparison of the translated ORFs revealed that the two $S$. platensis AHS isoenzymes are $66 \%$ identical. The deduced amino acid sequences of ilvX and ilv $W$ are respectively $70 \%$ and $61 \%$ identical with that of Synechococcus PCC 7942; $48 \%$ and $50 \%$ with that of ilvG; $42 \%$ and $44 \%$ with that of $i l v B$; and $46 \%$ and $44 \%$ with that of ilvI.

Haughn et al. (1988) have shown that in $A$. thaliana, a single $C$ to $T$ transition within codon 197 , resulting in proline being replaced by serine, is responsible for the mutant chlorsulphuron-resistant phenotype. Lee et al. (1988) have also shown that in the $N$. tabacum gene encoding AHS, the equivalent proline may be replaced by glutamine or alanine to give resistant forms of the enzyme. As shown in Fig. 2, motifs homologous to those of Brassica napus, $A$. thaliana and $N$. tabacum (AITGQVPRRMIGT; Wiersma et al., 1989) are also present in $i l v X$ and $i l v W$. It is noteworthy that proline is replaced by alanine in the $i l v X$ protein. Since $S$. platensis is resistant to $0.4 \mathrm{~mm}$-chlorsulphuron (data not shown), this may be due to the presence of a resistant form of AHS. This result was confirmed in the $E$. coli mutant complemented with $S$. platensis ilvX (data not shown).

\section{Transcriptional analysis}

RNA isolated from $S$. platensis, grown in minimal medium, was subjected to Northern blot analyses using, respectively, the $1.5 \mathrm{~kb} E c o \mathrm{RV}$ fragment from ilvX (nucleotides -215 to 1270 , Fig. $1 a$ ) and the $0.66 \mathrm{~kb}$ 
A MAGSGTTSTEKEETGAEFIVHELEQGGIKIVTAIFGGSILFVVDALS---QSTQIEHIL

B MN-.......-GAQWVVHALFAGGVWTVEGYFGGA IMFVYDALY - - - GGG-VEHLL

C MEM--.----LSGAEMVVESLIDQGVKQVEGYFGGAVLIIYDALH- - -TVGGIDHVL

D MQLQ-TVTIQQRATGAYALIDSLCQHGVKHIEGYFGGAILEIYDELHEAEAAGGEVGHIL

E MQLQ-TKIAAKRATGAERLIDSLKRHGVQHIEGYGGAILFIYDELYRAEAEGD-IQHIL

F MQ-D-QKQAVKEVTGAEALIDSLEFHGVQHIFGYFGGGNLFIYDEIYKAEQAGE-IKHYL

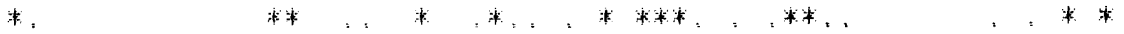

A AEHEGGAGEIAQGMAETDGKFAVMACGGEGATNLVTAIADAELDOIFLICITGQVFASM B CEHEQGAAMAAIGYAFATGKTGVCIATSGFGATNLITGLADALLUSIFVVAITGQVSAFE C VRHEQAAVHMAUGLAEATGEVGVUVTOGFGATNATTGIETAYMDSIFLVVLSGQVATSL D VEHEQEAVHAADAYCEATGEVGVFATSGEGATWLVTGIATAQMDSIFMVVVTGQVEETA E VEHEQGASHAADGVAEATGEVGVOFGTGGFGATNEVTGIATAHMDSIFMVI TTOQVAEFA $F$ VEHEQGAAHAADGYAETGKVGVCLATGFGATULVTGLATAYLDEVFVLAITGQVEFGA

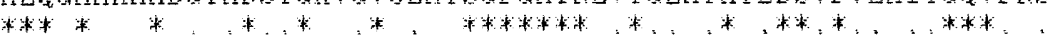

A IGTUAFQEVDTYGISIFITKHNYLVEHIEELFQVMSDAERIAQSGEFGEVWIDIFEDVGT B IGTEAEQEVDVLGLSLATTKHSELVQSLEELFRIMAEAFUVACSGEPGFVLVDIFKDIQL

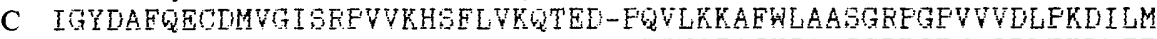
D IGTDAEQE-DIYGITLFIVKHEYVVRDFEDMAEIVAEAEHIAQGGEFGEVL IDVEXIVGT E IGTDAEQDSDIFGITLFIVKHSVVVEEFGMAEI VAEAEHIAGTGEFGFVL IDVEKIVGL F EGTDAEQEIDIEGITLFIVRHSYLVREFSELFEIVVEAFHLAMSGRFGPVLIDTEKDVGN

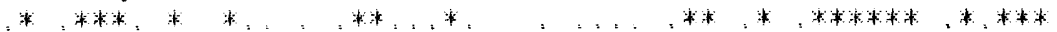

A AVEEIETQFAMAEKAAAFAEGEE - - - IEDAAAMINAAKRFVLYLGGGVIMAEA- - E B AGGULE--PWETTVENEVTEFHAE--..-VEQAFOMLARAGKFWLYVGGGYGMAQAVER C EANKLFYV-DFESVBMEYYNFTTTGHKGQIKRALQBVVAVKKFVVYVGGGAITAGCHQQ D E-DELYVPVAFGDIELFGYRFTT FGNFEQI-QAIALVKVAEFELLYVGGGAITAGAHAE E E--EEIYIFVWFGEVSLFGYEPTVKGNVRQINQAIKLIEEAERELMYVGGGAIOATAHAE

F A--QIUYIEVEFGEVEEVGYETEFGUERQI VQALOLISEATKFLLYVGGGAIMAGAHAE

A VEELAEKAOLFTTMTUMALGMLEKAHFLGLG--MLMHGVESTMY ILQEADLLIV-IGA B LEEFLAATKMEETCTLEGLGAVEADYFYYLG--MLGMHGTKAAMEAVQECDLL IAAVGA C LKETVEALNLFVVCSLMGLGAEEATHA-- QVLGMLGMHGTYEAMMTMHMAD-VIFAVGV

D LEAFAEREQLFVTTTLMGGAEUEFHELALG--MLGMMTAYANEAVSECULLT-AVGA

E IAELAELFQIFVTTTLHGKGSFDEKHLFSVG--MLGMHGTAYANEAVBECDELI-AVGA

F IAELSEREQIFVTSTLHGKGEDENHELGLOVOMLGMHGTAYANEAVMELDEVI-AVGV

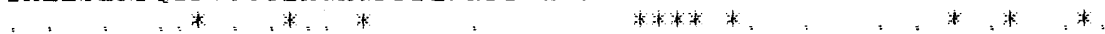

A EEDDEAIGETEQECFNA - KIIHVDIDRAELGK IKQFHVAIQADVDDVLADLIE-- LVEA

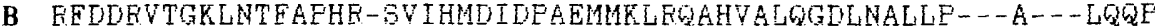

C EEVDETTUMLAKYCFN-ATVLHIDIDETEISKTVTAD IF IVGDAEQVLEQMLE--LLEQE

D ERDDEVTGKLDQEA-SKAQVIHVDIDFTSISKT VVFEVELVGDVRQVLNELLARA-DEQ

E BEDDFVTGKLDEEGTSEAKV IHIDIDFAEVGK NETEEVFIVGDVECVLIHLLERC-DE-

F BEDDEVAGTGDQEAHS-AKVIHIDIDFAEVGKHESTDVEIVGEVEQVLGDULQETYHWER

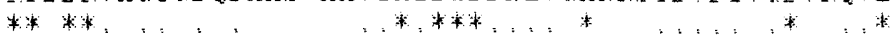

A QFEAEWHQLVADLQEE-----FFCFIFKACDELOHYGLTMAVACVDUWAITTTUVGQH B LNQYUWQQHCAQLEDEHSWE- - YDHFGDAIYAFL-.- - LIKQLODEKFADCVVTTDVGQH C SAHQFLDEIRDWWQGIEQWEAFQCLKYDTHSEK I KFGAVIETLWELTKGDAYUTSDVGQH D ISADDATETQEWLDEIAYWKEEYELQIYYYADVLSEQGVIHSLGE-AAFDAYETTDVGQH E IGDVGNDWQGSWLEFINBWEEDYELVVEGY SDSLOEQEVIAELGK-MAFDGYYTTDVGQH F RLSEWKFENGTDLNQL-- - FEFIPLTVEHFEDGISFQDGDWELSH-GCFTAEYTTDVGQH

A DMWTAQAYFLNEEFQWLTGGGLGTMGEGLFAATGAALANEDEKVLCESGDGOLMMNIQEM B QMWAAQH IAHTRFENE IESOGLGTMGEGLFAAVGAQVAEFWDTVVCISGDGSEMMNVQEL C QMEAALYYPEUREEEWIMGGGLGSHGEGLEAALGVKMAEEETVVCVTGIGSIQUNIQEL

D QMWAAQ-EIKNGFEAW I SSAGLGTMGFGMFAAMGAQMALFDDTY ICVAGDASILMNIQEL

E OMWAAQ-FLQWGEFQWISSAGLGTMGIGIEAMGAKVALEESQVIOIAGDASVQMNIQEL

F GMWAGQ-FVQWGEERWMTSGGLGTMGYGLFAAVGVKVAHFHETVTCISGDGSEQMMMQEL

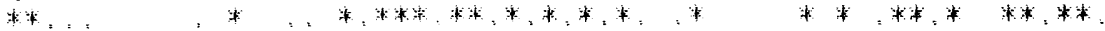

A ATASENQLDVKI ILMNUEALGLVHQGQDLFYEQVVAATY-GKINEMQIAAGEGLETCD B GTVKEKGLFLQIVLLDWQELGMVEQWQQLEFOEFYSETTLT-DNEDELMLABAEGIHGQH

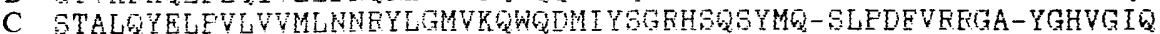

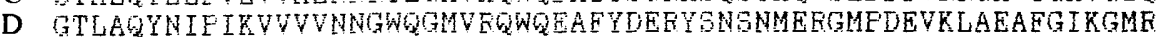

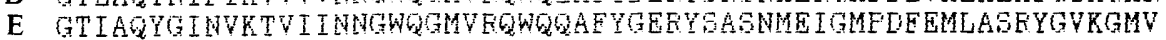

F GTIAGYGIGVKVI ILNWWWGMVEWWQHMEYWDEYATNLEDGTEEFAELADVYGLEAMA

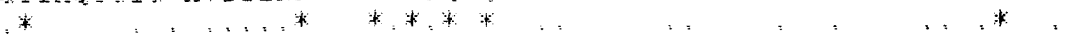



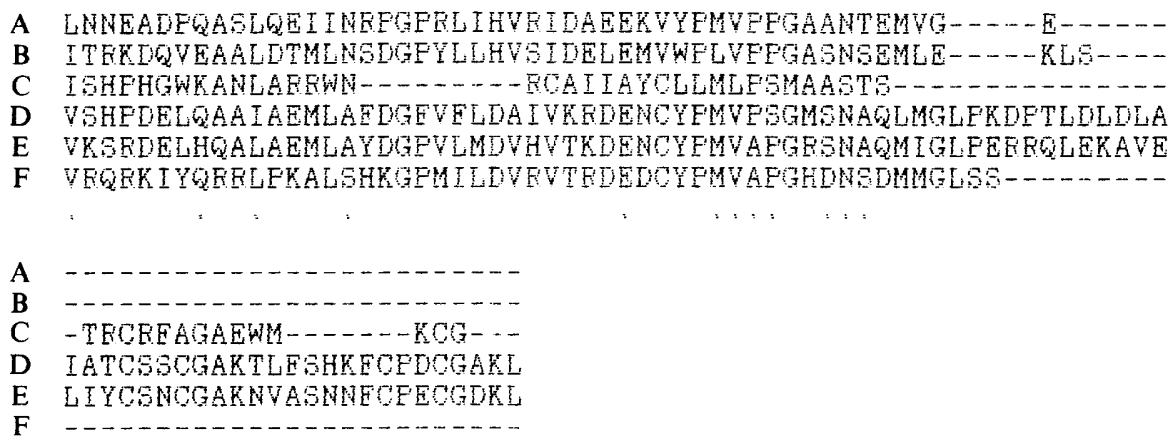

Fig. 2. Comparison of some known AHS amino acid sequences. The deduced amino acid sequences of genes encoding AHS are shown for: A, E. coli isoenzyme I; B, E. coli isoenzyme II ; C, E. coli isoenzyme III; D, Synechococcus PCC 7942 enzyme; E, Spirulina platensis isoenzyme I; F, S. platensis isoenzyme II. The overlined sequences indicate blocks of similarity associated with sulphonylurea-resistant phenotypes. Identical residues are indicated by asterisks, and conservative amino acid substitutions by dots. Dashes represent gaps inserted to optimize the protein alignment.

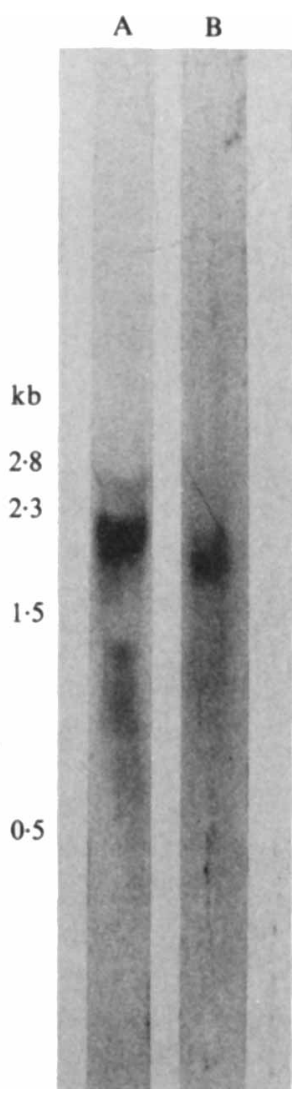

Fig. 3. Northern blot analysis of ilvX and ilvW transcripts in $S$. platensis. Total RNA extracted from $S$. platensis cells grown in minimal medium was fractioned on formaldehyde-agarose gel, transferred to a Hybond- $\mathrm{N}^{+}$filter and hybridized with the $1.5 \mathrm{~kb} E c o \mathrm{RV}$ fragment from $i l v X(\mathrm{~A})$ and the $0.66 \mathrm{~kb} E c o \mathrm{RV} /$ HaeIII fragment from $i l v W(\mathrm{~B})$ probes.

EcoRV/HaeIII fragment from ilvW (nucleotides 580 to 1240 , Fig. $1 b$ ) as internal probes (Fig. 3 ). The preponderant message species was about $2 \cdot 15 \mathrm{~kb}$ for ilv $X$ and
$1.95 \mathrm{~kb}$ for $i l v W$, corresponding roughly to the coding length required for the genes $(1836 \mathrm{~kb}$ for $i l v X$ and $1737 \mathrm{~kb}$ ilvW, Fig. 1).

The ilvBN and ilvGM operons are both regulated by transcription attenuation (Hauser \& Hatfield, 1983; Lawther \& Hatfield, 1980). The presence of a leader region (encoding a polypeptide rich in branched-chain amino acids) was not apparent in the $5^{\prime}$ flanking sequences of either ilvX or $i l v W$. Analysis of the $5^{\prime}$ noncoding region of ilvX and ilv $W$ disclosed putative promoter sequences homologous to known cyanobacterial promoters (Tandeau de Marsac \& Houmard, 1987). Some hypothetical -10 and -35 regions of ilv $X$ have resemblances to the $E$. coli consensus promoter sequences (TTGACA, TATAAT). This could be an explanation for the difference between the two $S$. platensis genes in the complementation of the $E$. coli mutant (Riccardi et al., 1991). To overcome this problem we are trying to place the ilv $W$ coding region under the control of an $E$. coli promoter. The ability to express this cyanobacterial enzyme in $E$. coli offers the opportunity to take advantage of the fast growth of $E$. coli cells to study a protein whose analysis is difficult in the organism of origin, essentially due to its high lability and to the presence of large amounts of pigments. It is noteworthy that the $5^{\prime}$ flanking regions of $i l v X$ and $i l v W$ contain, near a hypothetical -10 region, an inverted-repeat sequence that could play a role in the expression of these genes (operator?). Downstream from the $S$. platensis ilv $X$ and ilvW genes are, respectively, $30 \mathrm{bp}$ and $28 \mathrm{bp}$ sequences (underlined converging arrows in Fig. 1) which can code for an RNA capable of forming a stable stem and loop structure. The free energy of formation of such a conformation from these sequences is approximately $-25.8 \mathrm{kcal} \mathrm{mol}^{-1}(1 \mathrm{cal}=4.184 \mathrm{~J})$ in $i l v X$ and $-22.7 \mathrm{kcal}$ $\mathrm{mol}^{-1}$ in ilvW. Whether these structures act as a transcription terminator and/or as a barrier against 
$3^{\prime}$ exonuclease degradation remains to be determined. The nucleotide sequences of $i l v X$ and $i l v W$ provide the starting point for further studies on the regulation of cyanobacterial AHS.

The authors would like to thank Professor M. De Felice for helpful advice.

The present work was supported by MURST $60 \%$ and CNR Progetto Finalizzato PB no. 90.01304.14.

\section{References}

Barak, Z., Cal vo, J. M. \& Schloss, J. V. (1988). Acetolactate synthase isozyme III from Escherichia coli. Methods in Enzymology 166, 455458.

ChalefF, R. S. \& Mauvais, C. J. (1984). Acetolactate synthase is the site of action of two sulfonylurea herbicides in higher plants. Science 224, 1443-1445.

De Felice, M., Lago, C. T., Squires, C. M. \& Calvo, J. M. (1982). Acetohydroxy acid synthase isoenzymes of Escherichia coli K12 and Salmonella typhimurium. Annales de Microbiologie 133A, 251-256.

Eoyang, L. \& Silverman, P. M. (1988). Purification and assays of acetolactate synthase I from Escherichia coli K12. Methods in Enzymology 166, 435-445.

Falco, S. C. \& Dumas, K. D. (1985). Genetic analysis of mutants of Saccharomyces cerevisiae resistant to the herbicide sulfometuron methyl. Genetics 109, 21-35.

Falco, S. C., Dumas, K. D. \& Livak, K. J. (1985). Nucleotide sequence of the yeast ILV2 gene which encodes acetolactate synthase. Nucleic Acids Research 13, 4011-4027.

Forlani, G., Riccardi, G., De Rossi, E. \& De Felice, M. (1991). Biochemical evidence for multiple forms of acetohydroxy acid synthase in Spirulina platensis. Archives of Microbiology 155, 298-302.

FriedBerG, D. \& SEIJFFERS, J. (1990). Molecular characterization of genes coding for wild-type and sulfonylurea-resistant acetolactate synthase in the cyanobacterium Synechococcus PCC7942. Zeitschrift für Naturforschung 45c, 538-543.

Haughn, G. W., Smith, J., Mazur, B. \& Somerville, C. (1988), Transformation with a mutant Arabidopsis acetolactate synthase gene renders tobacco resistant to sulfonylurea herbicides. Molecular and General Genetics 21, 266-271.

HAUSER, C. A. \& HATFIELD, G. W. (1983). Nucleotide sequence of the ilvB multivalent attenuator of Escherichia coli K12. Nucleic Acids Research 11, 127-139.

Higgins, D. G. \& Sharp, P. M. (1989). Clustal: a package for performing multiple sequence alignments on a microcomputer. Gene $73,237-244$

KozAK, M. (1983). Comparison of initiation of protein synthesis in Procaryotes, Eucaryotes, and organelles. Microbiological Reviews 47, $1-45$

LANG, B. F. \& BURGER, G. (1986). A collection of programs for nucleic acid and protein analysis, written in FORTRAN 77 for IBM-PC compatible microcomputers. Nucleic Acids Research 14, 455-465.

LAWTHER, R. P. \& HATFIELD, G. W. (1980). Multivalent translation control of transcription termination at the attenuator of the ilvGEDA operon of Escherichia coli K-12. Proceedings of the National Academy of Sciences of the United States of America 77, 1862-1866.

Lawther, R. P., Calhoun, D. M., Adams, C. W., Hauser, C. A., Gray, J. \& Hatfield, G. W. (1981). Molecular basis of valine resistance in Escherichia coli K12. Proceedings of the National Academy of Sciences of the United States of America 78, 922-925.

Lee, K. Y., Townsend, J., Tepperwan, J., Black, M., ChuI, C. F., Mazur, B., DunSMUIR, P. \& BedBroOK, J. (1988). The molecular basis of sulfonylurea herbicide resistance in tobacco. EMBO Journal 7, 1241-1248.

Maniatis, T., Fritsch, E. F. \& SAmbrook, J. (1982). Molecular Cloning. A Laboratory Manual. Cold Spring Harbor, NY: Cold Spring Harbor Laboratory.

MaZUR, B. J., ChUI, C. F. \& SMITH, J. K. (1987). Isolation and characterization of plant genes coding for acetolactate synthase, the target enzyme for two classes of herbicides. Plant Physiology 85, $1110-1117$

RAY, T. B. (1984). Site of action of chlorsulfuron: inhibition of valine and isoleucine biosynthesis in plants. Plant Physiology 75, 827-831.

Reddy, K. J., BullerjahN, G. S., Sherman, D. M. \& Sherman, L. A (1988). Cloning, nucleotide sequence, and mutagenesis of a gene (irpA) involved in iron-deficient growth of the cyanobacterium Synechococcus sp. strain PCC7942. Journal of Bacteriology 170, 44664476.

RicCardi, G., Sora, S. \& Ciferri, O. (1981). Production of amino acids by analog resistant mutants of the cyanobacterium Spirulina platensis. Journal of Bacteriology 147, 1002-1007.

RicCardi, G., De Rossi, E. \& Milano, A. (1989). Amino acid biosynthesis and its regulation in cyanobacteria. Plant Science 63, 135-151.

Riccardi, G., Milano, A., De Rossi, E.;'Malfatti, C. \& De Felice, M. (1990). Amino acid biosynthesis in Spirulina. In Biosynthesis of Branched Chain Amino Acids, pp. 193-197. Edited by Z. Barak, D. M. Chipman \& J. V. Schloss. Weinheim: Verlag Chemie and Balaban Publishers.

Riccardi, G., De Rossi, E., Milano, A., Forlani, G. \& De Felice, M. (1991). Molecular cloning and expression of Spirulina platensis acetohydroxy acid synthase genes in Escherichia coli. Archives of Microbiology 155, 360-365.

SANGer, F., Nicklen, S. \& Coulson, A. R. (1977). DNA sequencing with chain-terminating inhibitors. Proceedings of the National Academy of Sciences of the United States of America 74, 5463-5467.

Schloss, J. V. \& VAN DYK, D. E. (1988). Acetolactate synthase isozyme from Salmonella typhimurium. Methods in Enzymology 166, 445-454.

Shaner, D. L., Anderson, P. C. \& Stidham, M. A. (1984) Imidazolinones: potent inhibitors of acetohydroxy acid synthase. Plant Physiology 76, 545-546.

Shine, J. \& Dalgarno, L. (1974). The $3^{\prime}$ terminal sequence of Escherichia coli $16 \mathrm{~S}$ ribosomal RNA : complementarity to nonsense triplets and ribosome binding sites. Proceedings of the National Academy of Sciences of the United States of America 71, 1342-1346.

Squires, C. H., De Felice, M., DevereuX, J. \& Calvo, J. M. (1983) Molecular structure of $i l v \mathrm{IH}$ and its evolutionary relationship to $i l v \mathrm{G}$ in Escherichia coli K12. Nucleic Acids Research 11, 5299-5313.

TANDEAU De Marsac, N. \& Houmard., J. (1987). Advances in cyanobacterial molecular genetics. In The Cyanobacteria, pp. 251295. Edited by P. Fay \& C. Van Baalen. Amsterdam: Elsevier.

Tomioka, N. \& SugiURA, M. (1983). The complete nucleotide sequence of a 16S ribosomal RNA gene from a blue-green alga, Anacystis nidulans. Molecular and General Genetics 191, 46-50.

UMBARGER, H. (1983). The biosynthesis of isoleucine and valine and its regulation. In Amino Acids: Biosynthesis and Genetic Regulation, pp. 245-265. Edited by K. M. Hermann \& R. L. Somerville. London: Addison Wesley.

WeK, R. C., Hauser, C. A. \& Hatfield, G. W. (1985). The nucleotide sequence of the ilvBN operon of Escherichia coli: sequence homologies of the acetohydroxy acid synthase isozymes. Nucleic Acids Research 13, 3995-4010.

Wiersma, P. A., Schmiemann, M. G., Condie, J. A., Crosby, W. L. \& MOLONEY, M. M. (1989). Isolation, expression and phylogenetic inheritance of an acetolactate synthase gene from Brassica napus. Molecular and General Genetics 219, 413-420.

Yasui, A., Takao, M., OIKaWa, A., Kiener, A., Walsh, C. T. \& Eker, A. P. M. (1988). Cloning and characterization of a photolyase gene from the cyanobacterium Anacystis nidulans. Nucleic Acids Research $16,4447-4463$ 